\title{
Orbital-Free Embedding Effective Potential in Analytically Solvable Cases
}

\author{
Andreas Savin and Tomasz A. Wesolowski
}

\begin{abstract}
The effective embedding potential introduced by Wesolowski and Warshel [J. Phys. Chem., 97 (1993) 8050] depends on two electron densities: that of the environment $\left(n_{B}\right)$ and that of the investigated embedded subsystem $\left(n_{A}\right)$. In this work, we analyze this potential for pairs $n_{A}$ and $n_{B}$, for which it can be obtained analytically. The obtained potentials are used to illustrate the challenges in taking into account the Pauli exclusion principle.
\end{abstract}

Keywords: Embedding potential - Density functional theory · Kinetic energy functional $\cdot$ Orbital-free embedding

\section{Introduction}

Computer simulation methods based on the idea of embedding are commonly used in numerical simulations of condensed matter: solids, liquids, interfaces, macromolecules (especially biomolecues), etc. The underlying concept behind the embedding strategy is very simple - one part of a total system is selected to be described by means of quantum mechanical descriptors such as orbitals whereas the remaining part of the whole system is considered as a source of some additional potential. Many strategies to construct embedding potentials are known in various areas of computational chemistry and computational material sciences. Usually, the embedding potential is postulated taking into account system-dependent parameters. Wesolowski and Warshel used the basic concepts of the Hohenberg-Kohn-Sham density functional theory [1, 2]: the reference system of non-interacting electrons, the functional of the kinetic energy in such a system

\footnotetext{
T.A. Wesolowski $(\bowtie)$

Department of Physical Chemistry, University of Geneva, Geneva, Switzerland, e-mail: tomasz.wesolowski@unige.ch
}

A. Savin

Laboratoire de Chimie Theorique, CNRS and Universite Pierre et Marie Curie (Paris VI), Paris, France, e-mail: andreas.savin@1ct.jussieu.fr 
$\left(T_{s}[n]\right)$, and exchange-correlation functional $\left(E_{x c}[n]\right)$, the external potential $v_{e x t}(\mathbf{r})$, and the exchange-correlation potential $v_{x c}[n](\mathbf{r})=\frac{\delta E_{x c}[n]}{\delta n(\mathbf{r})}$ to obtain one-electron equations for embedded orbitals (Eqs. (20) and (21) in Ref. [3]):

$$
\left[-\frac{1}{2} \nabla^{2}+v_{e x t}^{A}(\mathbf{r})+\int \frac{n_{A}\left(\mathbf{r}^{\prime}\right)}{\left|\mathbf{r}^{\prime}-\mathbf{r}\right|} d \mathbf{r}^{\prime}+v_{x c}\left[n_{A}\right](\mathbf{r})+v_{K S C E D}^{e m b}\left[n_{A}, n_{B}\right](\mathbf{r})\right] \varphi_{i}^{A}=\varepsilon_{i} \varphi_{i}^{A},
$$

where $v_{K S C E D}^{e m b}\left[n_{A}, n_{B}\right](\mathbf{r})$ is a system-independent expression for the embedding potential:

$$
\begin{aligned}
v_{K S C E D}^{e m b}\left[n_{A}, n_{B}\right](\mathbf{r})= & v_{e x t}^{B}(\mathbf{r})+\int \frac{n_{B}\left(\mathbf{r}^{\prime}\right)}{\left|\mathbf{r}^{\prime}-\mathbf{r}\right|} d \mathbf{r}^{\prime}+ \\
& \left.\frac{\delta E_{x c}[n]}{\delta n(\mathbf{r})}\right|_{n=n_{A}+n_{B}}-\left.\frac{\delta E_{x c}[n]}{\delta n(\mathbf{r})}\right|_{n=n_{A}}+ \\
& \left.\frac{\delta T_{s}[n]}{\delta n(\mathbf{r})}\right|_{n=n_{A}+n_{B}}-\left.\frac{\delta T_{S}[n]}{\delta n(\mathbf{r})}\right|_{n=n_{A}}
\end{aligned}
$$

and where $n_{A}$ denotes the density constructed from the embedded orbitals $\varphi_{i}$

$$
\sum_{i}^{\text {Nocc }} 2\left|\varphi_{i}\right|^{2}=n_{A}(r) .
$$

Throughout this chapter, equations are written in atomic units. For the sake of simplicity, equations are given for spin-compensated electron densities: hence the factor 2 in Eq. (3). The acronym KSCED stands for the Kohn-Sham equations with constrained electron density and is used to distinguish the two effective potentials expressed as density functionals: the one in the considered one-electron equations, which involves an additional constraint (see Eq. (5) below), from that in the KohnSham equations.

The above expression for the embedding potential, which was given explicitly in Eq. (3) of Ref. [4], shows clearly that except for $v_{e x t}^{B}(\mathbf{r})$ the position dependency of every other term in $v_{K S C E D}^{e m b}\left[n_{A}, n_{B}\right](\mathbf{r})$ is determined by the position dependency of $n_{A}$ and $n_{B}$. The symbol $v_{t}^{\text {nadd }}(\mathbf{r})$ will be used throughout this chapter the last two terms in Eq. (2), i.e., for the difference:

$$
v_{t}^{\text {nadd }}\left[n_{A}, n_{B}\right](\mathbf{r})=\left.\frac{\delta T_{s}[n]}{\delta n(\mathbf{r})}\right|_{n=n_{A}+n_{B}}-\left.\frac{\delta T_{s}[n]}{\delta n(\mathbf{r})}\right|_{n=n_{A}},
$$

arising from the fact that the functional $T_{s}[n]$ is not additive (see also the subsequent sections).

The embedding potential given in Eq. (2) was obtained by requiring that the ground-state energy of the total system including both the investigated subsystem 
described by means of Kohn-Sham orbitals and the environment is stationary for a given choice for $n_{B}$ which is not optimized (frozen). For this reason, we refer colloquially to methods using Eq. (2) as frozen density functional theory (FDFT) [3], frozen density embedding [5], etc. The derivation of Eq. (1) given in Ref. [3] (see also Ref. [6]) provided a new interpretation of embedding methodology in numerical simulations. Embedding calculations can be seen as the constrained optimization problem with the following weak constraint imposed on the total density $n$ :

$$
C[n] \geq 0
$$

where $C[n]=\min \left(n-n_{B}\right)$ and $n_{B}$ is the component of the electron total electron density which is not subject to optimization.

Note that the most common constraints in Euler-Lagrange equation take the form $C[n]=0$, where $C[n]$ is some density functional. For instance, the constraint $C[n]=\int n(\mathbf{r}) d \mathbf{r}-N=0$ is used in the derivation of the Kohn-Sham equations. Additional constraints expressed as $C[n]=0$ are also used in some computational schemes such as the procedure to generate diabatic electronic states for the evaluation of the rate of the electron-transfer reaction [7].

The results of a partial optimization of the total electron density in which $n_{B}(\mathbf{r})$ is frozen depend on the choice made for $n_{B}(\mathbf{r})$. Unless also $n_{B}$ is included in the optimization process (see for instance the $f$ reeze-and-thaw procedure of Ref. [4]), such partial minimization might lead to the total density which differs from the true ground-state electron density of the whole system, $n_{o}$. If $n_{B}$ is chosen to be such that $n_{0}(\mathbf{r})-n_{B}(\mathbf{r})<0$ in some domains, the density, $n_{A}$, obtained from Eqs. (1) and (3) cannot be equal to the complementary density $n_{o}-n_{B}$. Note that non-negativity of the complementary density for any given $n_{B}$ cannot be verified a priori. Moreover, the densities $n_{A}$ obtained from Eqs. (1) and (3) are pure-state non-interacting v-representable by construction. It is also not possible a priori to tell whether the density $n_{o}-n_{B}$ belongs to this class. These concerns can be avoided here because $v_{t}^{\text {nadd }}\left[n_{A}, n_{B}\right]$ is a functional of two electron densities and it is a well-defined quantity regardless $n_{A}+n_{B}, n_{A}$, and $n_{B}$ are ground-state densities of some non-interacting systems or not. Note that $v_{t}^{\text {nadd }}\left[n_{A}, n_{B}\right]$ is defined using the functional $T_{S}[n]$ for which extension exists (see Definitions and Notations section).

The embedding potential of Eq. (2) has been used as the basis for various computational methods. In most of our own numerical simulations (see for instance Refs. [8-11]), we use Eq. (1), and the partitioning of the total effective potential into its environment-free and embedding components is rather a technical issue. The orbital-free effective embedding potential given in Eq. (2) has been also used outside the domain for which it was derived, i.e., in combination with wave-function-based methods [12-14]. A detailed analysis of such a pragmatic combination of different treatment of the electron-electron interactions in the embedding potential and in the embedded component of the total electron density given in Ref. [15] revealed that it leads to double counting of significant energy contributions if Hartree-Fock method is used in combination with embedding potential given in Eq. (2). The same analyses 
showed that the magnitude of this double counting can be reduced or even entirely eliminated if the embedded system is described by means of a multi-determinantal "wavefunction." More recently, it was demonstrated that Eq. (2) provides the exact form of the potential to be used if the embedded object is described by means of one-particle reduced density matrices and the corresponding functional [16].

It is worthwhile to recall here that the position dependency of $v_{t}^{\text {nadd }}(\mathbf{r})$ is not explicit but it originates from the inhomogeneity of the densities $n_{A}$ and $n_{B}$. For the same potential, understood as a functional of $n_{A}$ and $n_{B}$, the symbol $v_{t}^{\text {nadd }}\left[n_{A}, n_{B}\right](\mathbf{r})$ or just $\left(v_{t}^{\text {nadd }}\left[n_{A}, n_{B}\right]\right)$ will be used. Such a distinction is of key importance for practical applications of Eq. (1) as they require the use of approximants to the functional $v_{t}^{\text {nadd }}\left[n_{A}, n_{B}\right]$. Moreover, the use of Eq. (1) in the more general framework of linear-response strategy for excited states involves functional derivatives of $v_{t}^{\text {nadd }}\left[n_{A}, n_{B}\right]$ with respect to $n_{A}[10,17]$.

In order to derive analytic forms of approximants to $v_{t}^{\text {nadd }}\left[n_{A}, n_{B}\right]$, various strategies are possible. A straightforward one relies on some known approximants to $T_{s}[n]$ which are used to derive analytic expression for $v_{t}^{\text {nadd }}\left[n_{A}, n_{B}\right][18,12,19]$. Such a strategy is based on the assumption that a reasonable approximant for $T_{S}[n]$ leads to a reasonable approximant to $v_{t}^{\text {nadd }}\left[n_{A}, n_{B}\right]$. Our dedicated studies on the relation between such approximants [18,20-22] showed that this assumption is not founded at least for the most common approximants. A more refined strategy relies on the direct analysis of the quality of electron density obtained from a given approximant to $v_{t}^{\text {nadd }}\left[n_{A}, n_{B}\right][18,20-22]$ rather than on the performance of the parent approximant to $T_{s}[n]$. Note that in calculations based on the Wesolowski-Warshel embedding formalism the absolute values of $T_{s}[n]$ obtained from an approximant to this functional are not needed. This strategy lead us to the GGA97 approximant [21] which was chosen as the remedy for erratic results obtained using the second-order gradient expression for $T_{S}[n]$ [18]. Finally, approximants to $v_{t}^{\text {nadd }}\left[n_{A}, n_{B}\right]$ can be derived using exact properties of this functional as guidelines or imposed conditions. This strategy uses such pairs of $n_{A}$ and $n_{B}$ for which the exact dependence of $v_{t}^{\text {nadd }}$ on position is available. For instance, the analysis of the behavior of $v_{t}^{\text {nadd }}\left[n_{A}, n_{B}\right]$ for $n_{A} \rightarrow 0$ and $\int n_{B} d \mathbf{r}=2$ led us to a new approximant referred to as NDSD (non-decomposable approximant using second derivatives) [23] superior in accuracy to the GGA97 one. It is useful to recall here that a "shortcut strategy" can be applied also in practice. It consists of using some analytic expression for $v_{t}^{\text {nadd }}(\mathbf{r})$ which depends explicitly on position [24]. This way of overcoming flaws of existing approximants to $v_{t}^{\text {nadd }}\left[n_{A}, n_{B}\right]$ proceeds without constructing any new approximant to $v_{t}^{\text {nadd }}\left[n_{A}, n_{B}\right]$.

The principal objective of this work is to obtain the exact form of $v_{t}^{\text {nadd }}(\mathbf{r})$ for some cases (choices $n_{A}$ and $n_{B}$ ). The considered cases make it possible not only to obtain $v_{t}^{\text {nadd }}(\mathbf{r})$ by means of the analytic inversion but also to illustrate the challenges to account for the Pauli exclusion principle by means of a multiplicative potential which is the functional of two densities, $v_{t}^{\text {nadd }}\left[n_{A}, n_{B}\right]$. In this work, no approximant to $v_{t}^{\text {nadd }}\left[n_{A}, n_{B}\right]$ is constructed, but the obtained shapes of $v_{t}^{\text {nadd }}(\mathbf{r})$ are to be used as guidelines for constructing approximants to $v_{t}^{\text {nadd }}\left[n_{A}, n_{B}\right]$ in the future works. 
It is worthwhile to point out at this point that this work uses extensively the inversion technique to obtain the external potential in a system of non-interacting electrons (Kohn-Sham system) which yield a given arbitrarily chosen target electron density. Finding numerically the potential associated with a given arbitrarily chosen target electron density is a well-known issue in density functional theory [25-27] and was even used recently [28] for obtaining the embedding potential in an alternative way to that given in Eq. (1). In this work, we analyze specific systems for which the inversion can be made analytically. The analytical inversion applied here cannot be seen, however, as an alternative to the numerical inversion techniques.

\section{Definitions and Notations}

The key quantity analyzed in this work is the potential $v_{t}^{\text {nadd }}(\mathbf{r})$ which is defined as the difference between the functional derivatives of the functional $T_{s}[n]$ at two different densities, $n$. The kinetic energy obtained in Kohn-Sham calculations provides the numerical value of the functional $T_{s}[n]$ defined in the Levy's constrained search [29]:

$$
T_{S}[n]=\min _{\Psi \rightarrow n}\langle\Psi|\hat{T}| \Psi\rangle
$$

Such a definition requires that $n$ is pure-state non-interacting $v$-representable, i.e., it is a ground-state density of some non-interacting system. In the particular cases analyzed later in this work, the density $n_{A}+n_{B}$ belongs to this narrower class. Unfortunately, pure-state non-interacting $v$-representability of $n_{A}$ cannot be assured. Therefore, interpreting $v_{t}(\mathbf{r})$ as the functional $v_{t}\left[n_{A}, n_{B}\right]$ is justified only if either both the densities $n_{A}+n_{B}$ and $n_{A}$ are pure-state non-interacting $v$-representable or if the definition of $T_{s}[n]$ is extendable to a wider class. Owing to the Levy-Lieb $[30,31]$ extension of $T_{s}[n]$ for ensembles

$$
T_{s}[n]=\min _{\omega_{i}, \Psi_{i} \sum_{i} \omega_{i}\left|\Psi_{i}\right|^{2} \rightarrow n} \sum_{i} \omega_{i}\left\langle\Psi_{i}|T| \Psi_{i}\right\rangle
$$

we shall not be concerned with the restriction of pure-state non-interacting $v$-representability of the densities considered in this work. Throughout this work, the following convention concerning notation for electron density is used:

$n_{B}$ is this component of the electron density which is not represented by means of orbitals and which is not optimized,

$n_{A}$ denotes the density obtained from Eqs. (1) and (3),

$n$ is just the sum of $n_{A}$ and $n_{B}$,

$n_{o}$ is the exact total ground-state electron density. 


\section{Analytical Results}

\subsection{Choice of the System for Obtaining $v^{\text {nadd }}\left[n_{A}, n_{B}\right]$}

A fictitious four-electron spherically symmetric system for which the exact KohnSham potential would read $v_{K S}=-\frac{1}{r}$ is considered in this work. The identification of the external potential $v_{\text {ext }}(r)$ which would correspond to such simple form of the Kohn-Sham potential is of no concern for the present considerations. For the purposes of the present analyses, it is crucial that the two doubly occupied KohnSham orbitals have the known exact analytic form of the hydrogenic wavefunctions $1 s$ and $2 s$. Note that the considered Kohn-Sham potential is neither the exact nor a reasonable approximant to the Kohn-Sham potential for a beryllium atom. The model bears some resemblance to a model used for a different purpose (the excited state of a two-electron system) in Ref. [32], Eq. (9). Although the analytical form of the dependence of $v_{t}^{\text {nadd }}\left[n_{A}, n_{B}\right]$ on $n_{A}$ and $n_{B}$ is not obtained, the exact form of $v_{t}{ }^{\text {nadd }}(\mathbf{r})$ can be constructed analytically for various choices made for $n_{A}$ and $n_{B}$ in the considered fictitious system. Below, the construction of $v_{t}{ }^{\text {nadd }}(\mathbf{r})$ is outlined.

In this case, the total density of the Kohn-Sham system reads

$$
n_{o}(\mathbf{r})=2\left(\phi_{1 s}^{2}+\phi_{2 s}^{2}\right)
$$

and the Kohn-Sham potential is

$$
v_{K S}=-\frac{1}{r}
$$

Let us consider the following decomposition of $n_{o}$ into the $n_{A}$ and $n_{B}$ components which are obtained as the combinations of orbital densities:

$$
\begin{aligned}
& n_{A}(\mathbf{r})=2\left((1-w) \phi_{1 s}^{2}+w \phi_{2 s}^{2}\right), \\
& n_{B}(\mathbf{r})=2\left(w \phi_{1 s}^{2}+(1-w) \phi_{2 s}^{2}\right) .
\end{aligned}
$$

At $w=0$, the frozen density $-n_{B}-$ is that of the valence, the doubly occupied $2 s$ orbital.

At $w=1$, the frozen density $-n_{B}-$ is that of the core, i.e., that of the doubly occupied $1 s$ orbital.

As $w$ increases from 0 to 1 , the density $n_{B}$ evolves from that localized in the valence to that localized in the core.

In this work, the effective potential which leads to the complementary density $n_{A}(\mathbf{r})$ is constructed for several choices made for $w$ and consequently for $n_{B}$. Since the potential obtained in this way has also the form given in Eqs. (1) and (2), 
$v_{t}^{\text {nadd }}(\mathbf{r})$ can be subsequently obtained. The subsequent sections concern the evolution of $v_{t}^{\text {nadd }}(\mathbf{r})$ as $w$ changes.

\subsection{Case I: $n_{B}$ Taken as Valence Electron Density}

Let us first choose $w=0$, i.e., $n_{B}=2 \varphi_{2 s}{ }^{2}$. The optimal $n_{A}$ obtained from Eq. (1) with such a choice for $n_{B}$ equals the complementary density $n_{A}=2 \varphi_{1 s}{ }^{2}$ only if the potential $v_{t}^{\text {nadd }}(\mathbf{r})$ disappears (or it is constant). Indeed, if $v_{t}^{\text {nadd }}=0$, the effective potentials in either the Kohn-Sham equations for the total system or in Eq. (1) (for the chosen $n_{B}$ ) are the same. As a consequence, $\varphi_{1 s}$ and $\varphi_{2 s}$ are eigenvectors of either equations and their eigenvalues are ordered in the same way. The lowest eigenvalue corresponds to $\varphi_{1 s}$, whereas the $\varphi_{2 s}$ is the solution which corresponds to the excited state of the reference system of non-interacting electrons. Therefore, the ground-state orbital obtained from Eq. (1) is also the square root of the target density $n_{o}-n_{B}$ (modulo the phase factor). The Aufbau principle is not violated. It is worthwhile to notice that despite the fact that the overlap between two orbital densities $n_{A}=2 \varphi_{1 s}{ }^{2}$ and $n_{B}=2 \varphi_{2 s}{ }^{2}$ is non-zero, $v_{t}^{\text {nadd }}=0$ (or constant). The simplest approximant to $v_{t}^{\text {nadd }}\left[n_{A}, n_{B}\right]$ derived from local density approximation (i.e., Thomas-Fermi approximant for $T_{s}[n]$ ) leads to a non-negative $v_{t}^{\text {nadd }}\left[n_{A}, n_{B}\right]$ if the densities do overlap. This indicates a systematic flaw of this approximant.

\subsection{Case II: $n_{B}$ Taken as Valence Electron Density with Small Admixture of Core Electron Density}

Let us now consider the case when $w$ is very small, but non-zero, i.e., transfer a very small amount of the previous $n_{B}$ density from the valence shell to the core.

On the one hand, the density to be determined from Eq. (1) is now asymptotically determined by the $2 s$ density. As a consequence, its eigenvalue must be equal to that of the $2 s$ orbital, i.e., $-1 / 8$, in order to have a potential which asymptotically goes to zero.

On the other hand, the potential cannot be changed in an important way to yield essentially the same density as for $w=0$.

The situation reminds of that of going from the system with $N$ electrons to that of $N+1$ electrons: in order to satisfy both requirements, the potential will be essentially shifted by a "constant", $(-1 / 2)-(-1 / 8))$, thus shifting the eigenvalue [33-36]. This happens over all space, except for the asymptotic region, where the $\varphi_{2 s}{ }^{2}$ dominates. Coming into that region, the potential falls down, finally approaching 0 .

The potential can be explicitly calculated, by inverting Eq. (1). Let $v_{s}(r)$ denote the whole potential in this equation. This potential can be expressed as $v_{K S}[n](r)+$ $v_{t}^{\text {nadd }}\left[n_{A}, n_{B}\right](r)$ (see Eq. (1)). For the considered system, $v_{s}(r)$ can be obtained as

$$
v_{s}(r)=\frac{1}{2} \frac{\nabla^{2} \sqrt{n-n_{B}}}{\sqrt{n-n_{B}}}+\text { constant },
$$




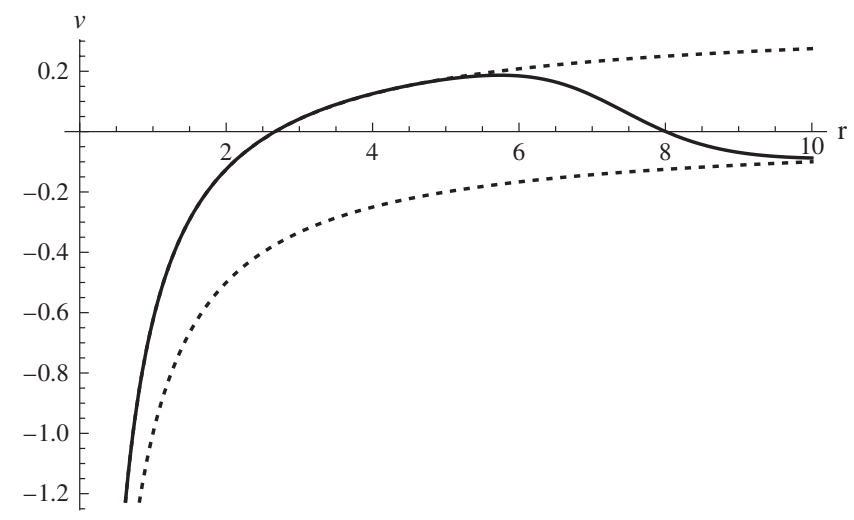

Fig. 1 The potential $(v(r)-b$ old line) obtained from Eq. (12) together with the Kohn-Sham potential $(-1 / r-$ lower dotted line) and the shifted Kohn-Sham potential $(-1 / r+1 / 2-1 / 8-u$ pper dotted line) obtained for $w=0.001$, i.e., $n_{A}=0.999 n_{1 s}+0.001 n_{2 s}$ and $n_{B}=0.001 n_{1 s}+0.999 n_{2 s}$

choosing the constant such that the potential goes to 0 when $r \rightarrow \infty$. Note that both $v_{s}(r)$ and $v_{K S}(r)$ are external potentials in a reference system of non-interacting electrons which are associated with different densities $-v_{s}(r)$ with $n-n_{B}$ whereas $v_{K S}(r)$ with $n$. In the chosen example, $v_{K S}(r)=-1 / r$. The difference between the two potentials obtained for $w=0.001$ is shown in Fig. 1

$v_{t}^{\text {nadd }}(\mathbf{r})$ is shown in Fig. 2 for the same $w=0.001$, together with $4 \pi r^{2} n_{A}(r)$. On the scale of the plot, the contribution of the $2 s$ density is not noticeable. For higher values of $w$, it appears as a shoulder or for even higher values of $w$ yields a second maximum in $4 \pi r^{2} n_{A}(r)$.

As the contribution of the $2 s$ density increases, the jump in $v_{t}^{\text {nadd }}(r)$ is further displaced toward the origin, as can be seen by comparing Figs. 1 and 3 which show $v(r)$ for $w=0.001$ and $w=0.01$, respectively.

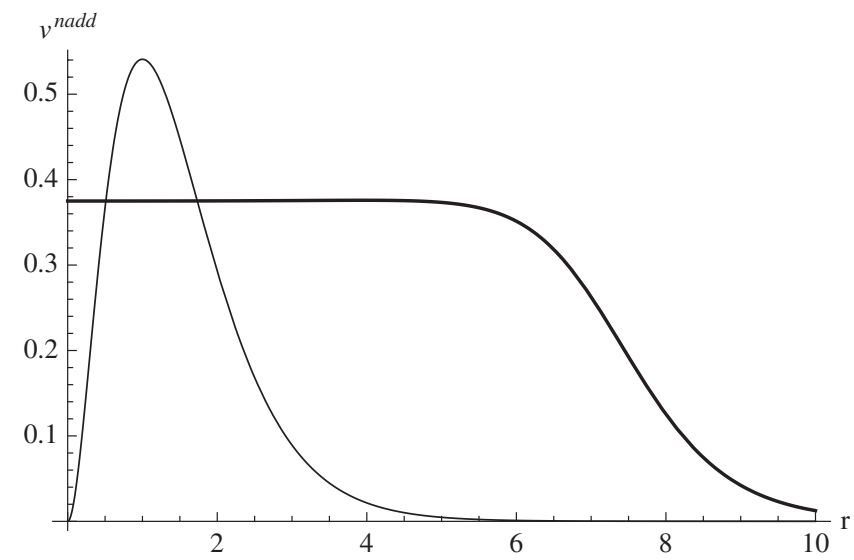

Fig. $2 v_{t}^{\text {nadd }}(r)$ (bold line) $-1 / r$, obtained for $w=0.001$, i.e., $n_{A}=0.999 n_{1 s}+0.001 n_{2 s}$ and $n_{B}=0.001 n_{1 s}+0.999 n_{2 s}$. The arbitrarily normalized plot of $4 \pi r^{2} n_{A}$ is also shown (thin line) 


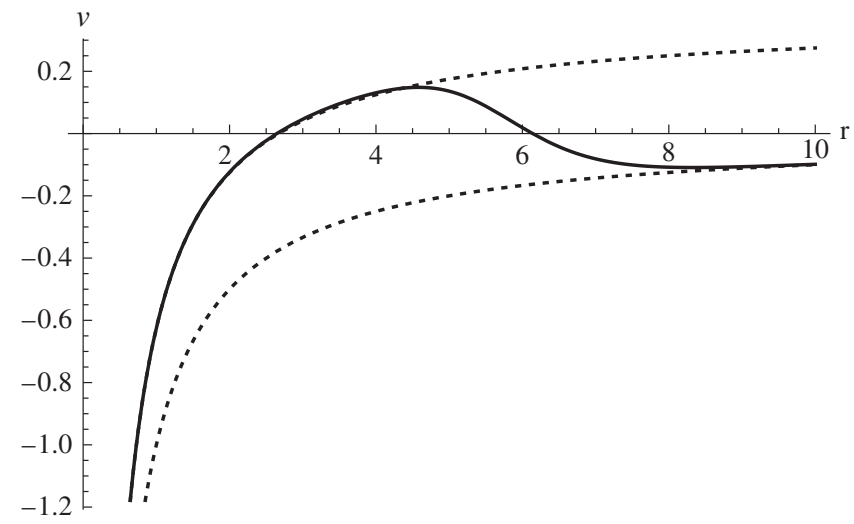

Fig. 3 The potential $(v(r)-$ bold line) obtained from Eq. (12) together with the Kohn-Sham potential $(-1 / r-$ lower dotted line) and the shifted Kohn-Sham potential $(-1 / r+1 / 2-1 / 8-u$ pper dotted line) obtained for $w=0.01$, i.e., $n_{A}=0.99 n_{1 s}+0.01 n_{2 s}$ and $n_{B}=0.01 n_{1 s}+0.99 n_{2 s}$

\subsection{Case III: $n_{B}$ Taken as Valence Electron Density with Noticeable Admixture of Core Electron Density}

As the contribution of the $2 s$ density to $n_{A}$ (i.e., the density to be determined from Eq. (1)) increases, a bump starts to be apparent in the plot of the inverted potential $v(r)$ (compare Figs. 3, 4, and 5).

The bump in $v(r)$ originates from the rapid variation of $v_{t}^{\text {nadd }}$ component (compare Figs. 4 and 5 with Figs. 6 and 7, respectively).

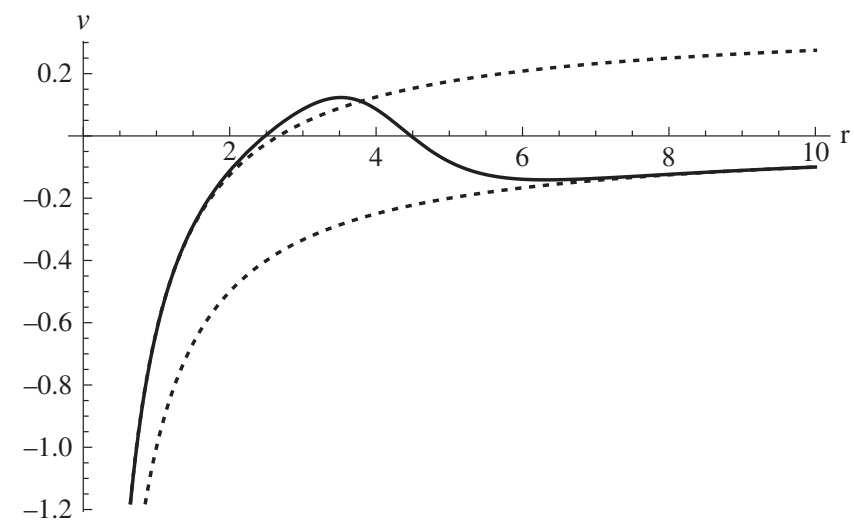

Fig. 4 The potential $(v(r)$ - bold line) obtained from Eq. (12) together with the Kohn-Sham potential $(-1 / r-$ lower dotted line) and the shifted Kohn-Sham potential $(-1 / r+1 / 2-1 / 8-u$ pper dotted line) obtained for $w=0.1$, i.e., $n_{A}=0.9 n_{1 s}+0.01 n_{2 s}$ and $n_{B}=0.1 n_{1 s}+0.9 n_{2 s}$ 


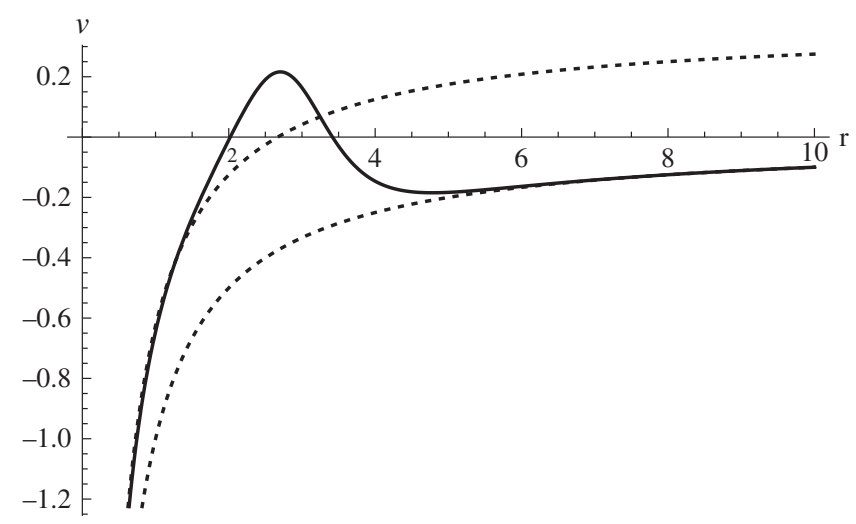

Fig. 5 The potential $(v(r)$ - bold line) obtained from Eq. (12) together with the Kohn-Sham potential $(-1 / r-$ lower dotted line) and the shifted Kohn-Sham potential $(-1 / r+1 / 2-1 / 8-u$ pper dotted line) obtained for $w=0.5$, i.e., $n_{A}=0.5 n_{1 s}+0.5 n_{2 s}$ and $n_{B}=0.5 n_{1 s}+0.5 n_{2 s}$

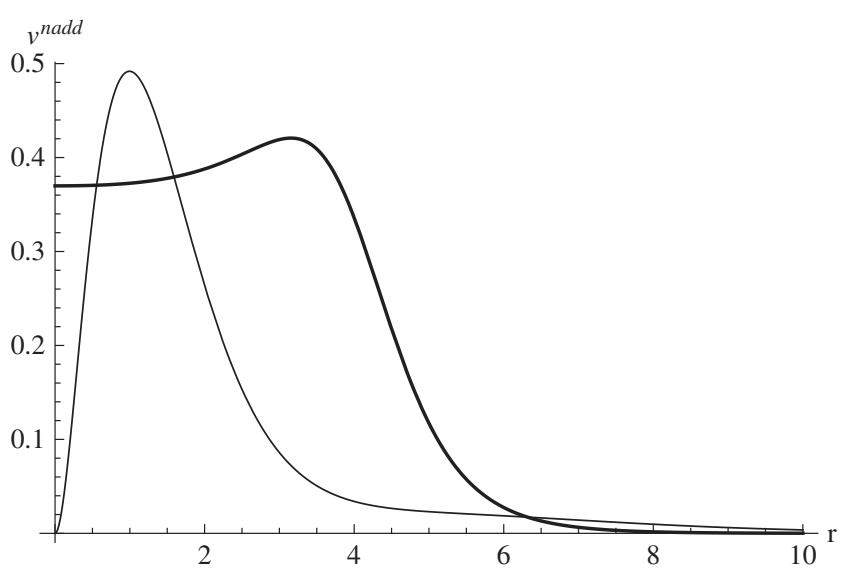

Fig. $6 v_{t}^{\text {nadd }}(r)$ (bold line) $-1 / r$, obtained for $w=0.9$, i.e., $n_{A}=0.9 n_{1 s}+0.1 n_{2 s}$ and $n_{B}=$ $0.1 n_{1 s}+0.9 n_{2 s}$. The arbitrarily normalized plot of $4 \pi r^{2} n_{A}$ is also shown (thin line)

\subsection{Case IV: $n_{B}$ Taken as Core Electron Density with Small Admixture of Valence Electron Density}

The bump in $v(r)$ (or in $v_{t}^{\text {nadd }}$ ) gets more and more pronounced as $w$ increases. Figs. 8 and 9 show $v(r)$ for $w=0.9$ and for $w=0.99$, respectively.

The origin of the dip in the density (and the bump in the potential needed to produce it) is clear: the contribution of the $2 s$ density to $n_{A}$ increases with $w$; at 100 per cent $2 s$ density, one even has $n_{A}(r=2)=0$. 


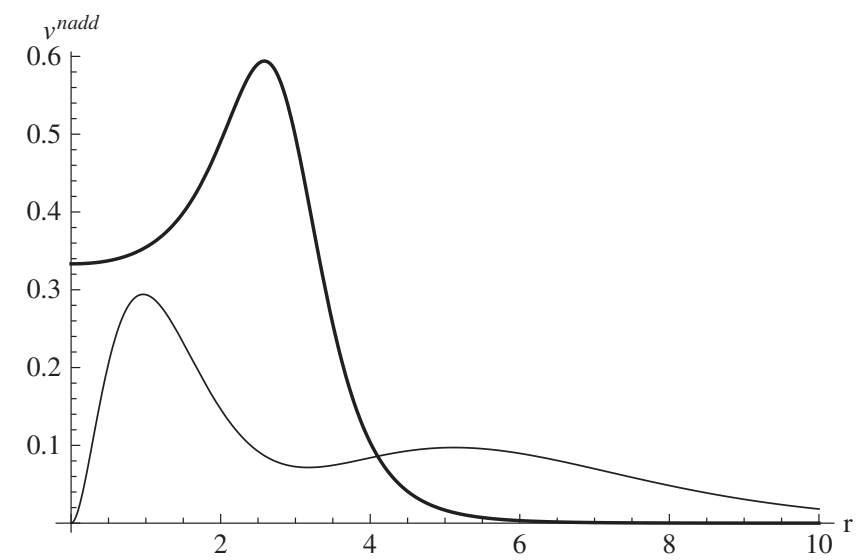

Fig. $7 v_{t}^{\text {nadd }}(r)$ (bold line) $-1 / r$, obtained for $w=0.5$, i.e., $n_{A}=0.5 n_{1 s}+0.5 n_{2 s}$ and $n_{B}=$ $0.5 n_{1 s}+0.5 n_{2 s}$. The arbitrarily normalized plot of $4 \pi r^{2} n_{A}$ is also shown ( $t$ hin line)

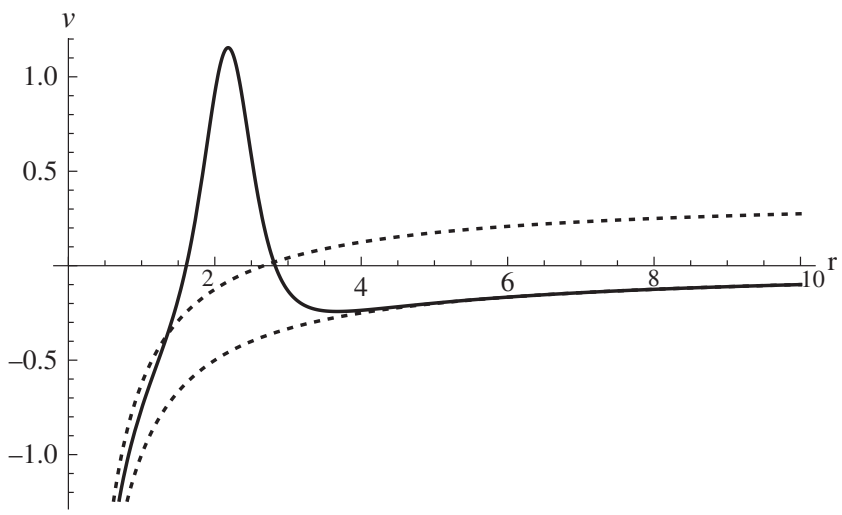

Fig. 8 The potential $(v(r)$ - bold line) obtained from Eq. (12) together with the Kohn-Sham potential $(-1 / r-$ lower dotted line) and the shifted Kohn-Sham potential $(-1 / r+1 / 2-1 / 8-u$ pper dotted line) obtained for $w=0.9$, i.e., $n_{A}=0.1 n_{1 s}+0.9 n_{2 s}$ and $n_{B}=0.9 n_{1 s}+0.1 n_{2 s}$

\subsection{Case V: $n_{B}$ Taken as Core Electron Density}

At the first sight, it seems that when $n_{B}$ is the core electron density $\left(n_{B}=2 \cdot 1 s^{2}\right)$, the density $n_{o}-n_{B}$ can be obtained from Eqs. (1) and (3) by putting $v_{t}^{\text {nadd }}(r)=0$, i.e., for $v(r)+v_{t}^{\text {nadd }}(r)=-1 / r$. Indeed, the orbital $2 s$ is one of the eigenfunctions in the equation $\left[-\frac{1}{2} \nabla^{2}-1 / r\right] \varphi=\varepsilon \varphi$. It is, however, not the lowest eigenvalue. For $v_{t}^{\text {nadd }}(r)=0$, Eqs. (1) and (3) would lead, therefore, to the total electron density $n_{A}+n_{B}=4 \cdot 1 s^{2}$ which would violate the Pauli exclusion principle. Satisfying the requirement that the ground-state orbital obtained from Eq. (1) yields such density $n_{A}$ that $n_{A}=n_{o}-n_{B}$ for $n_{B}$ being the core electron density must be reflected in the 


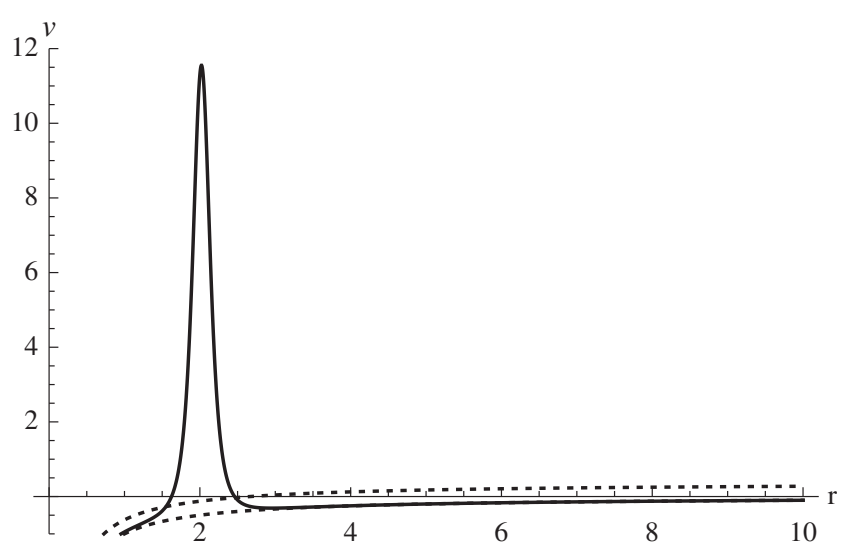

Fig. 9 The potential $(v(r)-$ bold line) obtained from Eq. (12) together with the Kohn-Sham potential $(-1 / r-$ lower dotted line) and the shifted Kohn-Sham potential $(-1 / r+1 / 2-1 / 8-u$ pper dotted line) obtained for $w=0.99$, i.e., $n_{A}=0.01 n_{1 s}+0.99 n_{2 s}$ and $n_{B}=0.99 n_{1 s}+0.01 n_{2 s}$

$v_{t}^{\text {nadd }}(r)$ component of the total potential. The solution to this paradox is provided by the existence of the bump which is transformed into a barrier that is infinitely high and vanishingly thin. This barrier can be seen as a change in the boundary conditions on the Schrödinger equation.

Figure 11 shows the appearance of the barrier-like character of $v_{t}^{\text {nadd }}(r)$ for $w=$ 0.999 .

A final question remains to be clarified. The integral of $n_{A}$ on each of the segments ( $r$ between 0 and 2 and from 2 to infinity, respectively) is a non-integer number. What is the wave function in such a case? In fact, in this case we have to deal with two spatial regions: the inner sphere and the outer spherical shell. The $2 s$ orbital is an eigenfunction of the Hamiltonian on both segments and the eigenvalue is, of course, the same $(-1 / 8)$. To obtain the ground state with the density given, we use the nodeless functions on each of the segments; next, we produce ensembles with weights equal to the integral of the $2 s$ orbital from 0 to 2 and from 2 to infinity ( 0.053 and 0.947 , respectively) and get the correct density.

$v_{t}^{\text {nadd }}(r)$ evolves thus from $v_{t}^{\text {nadd }}(r)=0$ at $w=0$ to a delta-like potential as $w$ approaches 1 . It is worthwhile to look more closely at the $w=0.5$ case. If $w=0.5$, the not-optimized component of the electron density, $n_{B}$, is exactly the same as the target electron density $n_{A}=n_{o}-n_{B}=1 / 2 n_{o}$. The equation $\left[-\frac{1}{2} \nabla^{2}+v(r)\right] \varphi=\varepsilon \varphi$, where $v(r)$ is the potential shown in Fig. 5, leads to such eigenfunction that $2 \varphi^{2}=n_{o} / 2$. It is worthwhile to notice that no orbital representation of the density $n_{B}$ has been used so far in our considerations. In the $w=0.5$ case, one can trivially represent also $n_{B}$ by means of an orbital given by the square root of $n_{B}=1 / 2 n_{o}$. Since $n_{A}=n_{B}$ the two "orbitals" are the same (module phase factor) and are obviously strongly non-orthogonal. Nevertheless, Eq. (1) still leads to the exact ground-state electron density for the total system. This example illustrates that the embedded orbitals obtained from Eq. (1) and orbitals obtained from some 

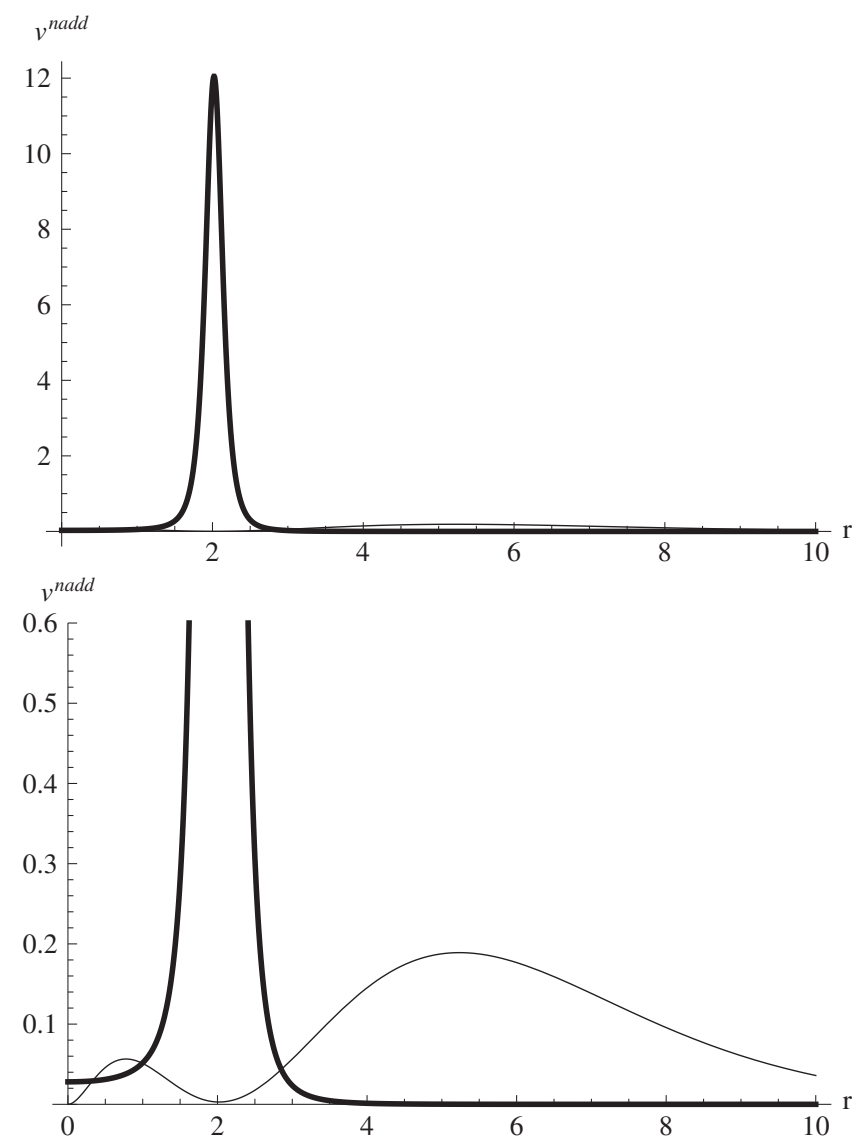

Fig. $10 v_{t}^{\text {nadd }}(r)$ (bold line) $-1 / r$, obtained for $w=0.9$, i.e., $n_{A}=0.9 n_{1 s}+0.1 n_{2 s}$ and $n_{B}=$ $0.1 n_{1 s}+0.9 n_{2 s}$. The arbitrarily normalized plot of the radial density, $4 \pi r^{2} n_{A}$, is also shown ( $t$ hin line). The $u$ pper and lower figures show the same potential at different ranges

reconstruction of the assumed density $n_{B}$ represent different objects which should not be confused with any wave function representation of the total system.

\section{Conclusions}

In the embedding formalism introduced by Wesolowski and Warshel [3], the total electron density is partitioned into two components. One of them is not optimized (frozen) and the other is subject to optimization. The optimized component is treated in a Kohn-Sham-like way, i.e., by means of a reference system of non-interacting electrons. The multiplicative potential in one-electron equations for embedded orbitals, Eq. (1) or Eqs. (20) and (21) of Ref. [3], differs from the Kohn-Sham 


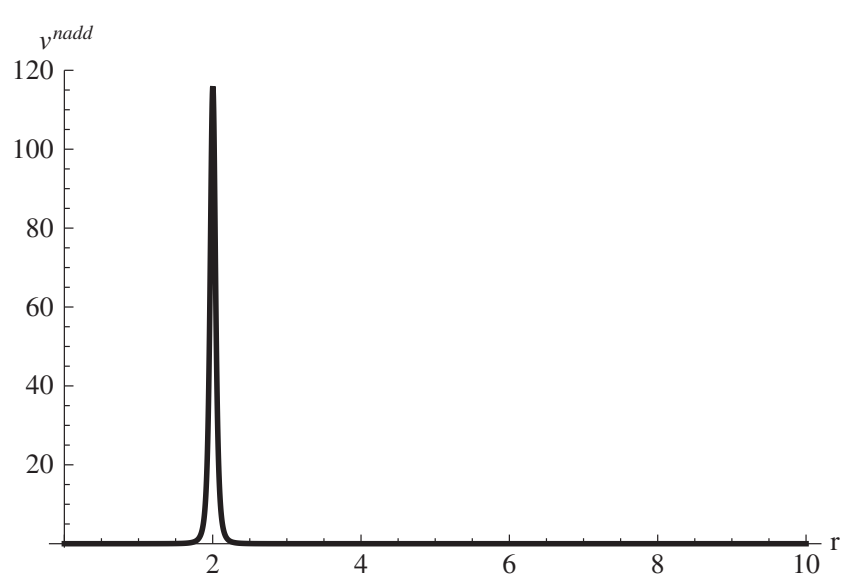

Fig. $11 v_{t}^{\text {nadd }}(r)$ (bold line) $-1 / r$, obtained for $w=0.999$, i.e., $n_{A}=0.999 n_{1 s}+0.001 n_{2 s}$ and $n_{B}=0.001 n_{1 s}+0.999 n_{2 s}$. The arbitrarily normalized plot of the radial density, $4 \pi r^{2} n_{A}$, is also shown (thin line)

potential by an additional term arising from non-additivity of the kinetic energy, $v_{t}^{\text {nadd }}(r)$. The question arises how such multiplicative potential takes into account the Pauli exclusion principle. In particular, if the non-optimized component of the total density coincides with the orbital density of the lowest lying Kohn-Sham orbital of the whole system, how to avoid that the complementary density obtained from Eq. (1) collapses on the not-optimized one?

A simple example is analyzed of the case when the exact effective potential in one-electron equations for embedded orbitals (Eqs. (20) and (21) of Ref. [3]) can be obtained analytically. The Kohn-Sham orbitals for the considered total system are the hydrogenic functions $1 s$ and $2 s$. For particularly partitioned total density of such system, in which the not-optimized component of the electron density is a mixture of $1 s$ and $2 s$ orbital densities, the exact potential in Eq. (1) can be obtained analytically. In the considered examples, the density $n_{A}$ obtained from Eq. (1) complements perfectly the chosen not-optimized one, $n_{B}$, so they add to the exact ground-state density. Depending on the choice for $n_{B}$ and $n_{A}$ resulting from it the following situations are observed:

- If $n_{A}$ is the density that is given by the lowest-lying orbital the Kohn-Sham potential for the whole system coincides with the effective potential in Eq. (1). No additional potential is needed $\left(v_{t}^{\text {nadd }}(r)=0\right)$.

- If $n_{A}$ is essentially given by the lowest-lying orbital, with only a small admixture of the higher-lying orbtials, the additional potential is mainly shifted in the region of interest; this shift aligns the orbital energy of the lower-lying orbital to that of the higher-lying one, allowing the mixing of the densities of different states. 
- As the contribution to $n_{A}$ due to the higher-lying level increases, a bump shows up in the nodal region of the corresponding orbital (density). The bump forbids electrons to enter the region close to the node of the high-lying orbital.

- If $n_{A}$ is the density of the high-lying orbital, the bump is transformed into an infinite barrier and an ensemble description has to be used for $n_{A}$. In this case, $v_{t}^{\text {nadd }}(r)$ becomes an infinitely high barrier of vanishing thickness.

Turning back to the issue of developing approximants to $v_{t}^{\text {nadd }}\left[n_{A}, n_{B}\right]$, i.e., representing the potential $v_{t}^{\text {nadd }}(\mathbf{r})$ as functional of the two electron densities without introducing any explicit position-dependency, the analytic results obtained in this work exposed rather serious difficulties if one would aim at some universal, system- and also partitioning-independent approximant to $v_{t}^{\text {nadd }}\left[n_{A}, n_{B}\right]$. For instance, the obtained shapes of the $v_{t}^{\text {nadd }}(\mathbf{r})$ indicate that this potential changes abruptly with minor modifications of $n_{A}$ and $n_{B}$. Moreover, examples were given where $v_{t}^{\text {nadd }}\left[n_{A}, n_{B}\right] \neq v_{t}^{\text {nadd }}\left[n_{B}, n_{A}\right]=$ const and the densities $n_{A}$ and $n_{B}$ do overlap and are far from uniform.

Acknowledgements The authors thank Prof. Pietro Cortona for helpful discussions. T. A. W. and A. S. acknowledge the support from the grants by Swiss National Research Foundation (Project 200020-116760) and ANR (Project 07-BLAN-0272), respectively.

\section{References}

1. P. Hohenberg, W. Kohn, Phys. Rev. B 136, 864 (1964)

2. W. Kohn, L. J. Sham, Phys. Rev. 140, A1133 (1965)

3. T. A. Wesołowski, A. Warshel, J. Phys. Chem. 97, 8050 (1993)

4. T. A. Wesolowski, J. Weber, Chem. Phys. Lett. 248, 71 (1996)

5. ADF 2003.01, SCM, Theoretical Chemistry, Vrije Universiteit, Amsterdam, The Netherlands, http://www.scm.com.

6. T. A. Wesolowski, in Computational Chemistry: Reviews of Current Trends, vol. X, ed. by J. Leszczynski (World Scientific, Singapore, 2006), p. 1

7. Q. Wu, T. Van Voorhis, J. Chem. Phys. 125, 164105 (2006)

8. T. A. Wesołowski, Chem. Phys. Lett. 311, 87 (1999)

9. M. Zbiri, M. Atanasov, C. Daul, J.-M. Garcia Lastra, T. A. Wesolowski, Chem. Phys. Lett. 397, 441 (2004)

10. T. A. Wesolowski, J. Am. Chem. Soc. 126, 11444 (2004)

11. M. Leopoldini, N. Russo, M. Toscano, M. Dulak, T. A. Wesolowski, Chem. Eur. J. 12, 2532 (2006)

12. T. Klüner, N. Govind, Y. A. Wang, E. A. Carter, J. Chem. Phys. 116, 42 (2002)

13. D. Lahav, T. Kluner, J. Phys. - Cond. Matt. 19, 226001 (2007)

14. C. R. Jacob, L. Visscher, J. Chem. Phys. 128, 155102 (2008)

15. T. A. Wesolowski, Phys. Rev. A 77, 012504 (2008)

16. K. Pernal, T. A. Wesolowski, Int. J. Quantum. Chem. 109, 2520 (2009)

17. M. Casida, T. A. Wesolowski, Int. J. Quantun Chem. 96, 577 (2004)

18. T. A. Wesolowski, J. Weber, Int. J. Quantum Chem. 61, 303 (1997)

19. M. Hodak, W. Lu, J. Bernholc, J. Chem. Phys. 128, 014101 (2008)

20. T. A. Wesolowski, H. Chermette, J. Weber, J. Chem. Phys. 105, 9182 (1996) 
21. T. A. Wesolowski, J. Chem. Phys. 106, 8516 (1997)

22. Y. A. Bernard, M. Dulak, J. W. Kaminski, T. A. Wesolowski, J. Phys. A 41, 055302 (2008)

23. J.-M. Garcia Lastra, J. W. Kaminski, T.A. Wesolowski, J. Chem. Phys. 129, 074107 (2008)

24. C. R. Jacob, S. M. Beyhan, L. Visscher, J. Chem. Phys. 126, 234116 (2007)

25. Q. Zhao, R. C. Morrison, R. G. Parr, Phys. Rev. A 50, 2138 (1994)

26. E. J. Baerends, Phys. Rev. Lett. 87, 133004 (2001)

27. W. Yang, Q. Wu, Phys Rev. Lett. 89, 143002 (2002)

28. O. Roncero, M. P. de Lara-Castells, P. Villarreal, F. Flores, J. Ortega, M. Paniagua, A. Aguado, J. Chem. Phys. 129, 184104 (2008)

29. M. Levy, Proc. Natl. Acad. Sci. USA 76, 6062 (1979)

30. M. Levy, Phys. Rev. A 26, 1200 (1982)

31. E. H. Lieb, Int. J. Quantum Chem. 24, 243 (1983)

32. J. P. Perdew, M. Levy, Phys. Rev. B 31, 6264 (1985)

33. J. P. Perdew, R. G Parr, M. Levy, J. L. Balduz Jr., Phys. Rev. Lett. 49, 1691 (1982)

34. D. J. Tozer, N. C. Handy, J. Chem. Phys. 109, 10180 (1998)

35. F. Colonna, A. Savin, J. Chem. Phys. 110, 2828 (1999)

36. F. Della Sala, A. Görling, Andreas, Phys. Rev. Lett. 89, 033003 (2002) 